\title{
Acoustic Beam Forming Using Ultrasonic Transducers
}

\author{
Wen-Kung Tseng \\ Graduate Institute of Vehicle Engineering, \\ National Changhua University of Education, Changhua City, Taiwan, R.O.C. \\ e-mail:andy007@cc.ncue.edu.tw
}

\begin{abstract}
Keywords: directional audible sound, ultrasound carrier, ultrasonic transducers, an optimization technique, uniform linear array.
\end{abstract}

\begin{abstract}
This paper evaluates the performance of acoustic beam forming using ultrasonic transducers. A directional audible sound can be generated by amplitude-modulating the ultrasound carrier with an audio signal, then transmitting it from an array of ultrasonic transducers. A novel method has been proposed in this paper to control the beam width of the main lobe and the level of the side lobe for the beam pattern by using an optimization technique. Furthermore, the weighting distribution of uniform linear array composed of eight transducers and the effect of different weightings on the spreading angle of the sound beam have been investigated through simulations in this study. The results show that the optimization method proposed in the paper can effectively control the beam width of the main lobe and the level of the side lobe for the audible sound.
\end{abstract}

\section{Introduction}

An array of ultrasonic transducers may be employed for generating the directional audible sound through the self-demodulation of finite-amplitude sound beams [1-5]. The construction of producing directional audible sound beams includes an ultrasonic transducer, a driving amplifier for the array, an AM modulator, a pure-tone oscillator for the carrier frequency and equalizer. An array of ultrasonic transducers may be called a parametric speaker regarding the application of the parametric array theory. The parametric speaker has been used for decades in underwater sonar applications for its highly directive response even at low frequencies [6]. The transducer array in parametric speaker systems may comprise a plurality of piezoelectric transducers or polyvinylidene fluoride (PVDF) film transducers. For simplicity, we can assume that the transducer is fed with two ultrasonic signals at closely frequencies $f_{1}$ and $f_{2}$. The resultant acoustic waves will be the waves with the frequencies of $f_{1}, f_{2}, f_{1} \pm f_{2}$, and higher order harmonics, etc. Among these new components, the high-frequency terms $f_{1}, f_{2}$ and $f_{1}+f_{2}$, etc. will be strongly attenuated in air and decay rapidly with increasing range from the speaker. The remaining difference frequency $f_{1}-f_{2}$ is produced due to the relatively low absorption of this term in air [5]. Moreover, this low-frequency sound, the audible sound, inherits the spatial characteristics of the primary waves, and its sharp directivity is the result of the acoustic nonlinearity. The audible sound is the secondary wave generated from the primary wave by a nonlinear effect, which has been reported for a few decades [6]. Since the first attempt to establish an 'audio spotlight' system [7], the development of a directional parametric speaker has attracted much attention.

Most of the works related to directional audible sound beam focused on investigating the directivity of the sound beam or the different transducer arrangement. There were only a few works on the beam width control in a parametric array [5,8]. Yang et al. proposed the weighted parametric array using Chebyshev window method for the transducer weightings to generate the directional audible sound [5]. However in this paper an optimization method is used to calculate the optimal transducer weightings for controlling the beam width of the main lobe and the level of the side lobes for the directional audible sound in vehicle. Also the beam steering performance has been investigated in this work. Some preliminary experiment has been carried out to validate the simulation results. 
The paper is organized as follows. First, the method of acoustic beam forming is described. Second, simulation results of controlling the beam width of the main lobe and the level of side lobes are presented. Finally the conclusions are made.

\section{Method of acoustic beam forming}

In this section the formulation for designing directional audible sound systems using an optimization technique is presented. The directional audible sound can be described by the Khokhlov-Zabolotskaya-Kuznetsov (KZK) equation which accounts for the combined effects of nonlinearity, absorption due to viscosity and heat conduction, and diffraction in finite-amplitude sound beam as follows $[5,9]$ :

$$
\frac{\partial^{2} p}{\partial z \partial \tau}=\frac{c}{2} \nabla_{\perp}^{2} p+\frac{\delta}{2 c^{3}} \frac{\partial^{3} p}{\partial \tau^{3}}+\frac{\beta}{2 \rho_{0} c^{3}} \frac{\partial^{2} p^{2}}{\partial \tau^{2}}
$$

where $p$ denotes the acoustic pressure, $z$ is the coordinate along the axis of the beam propagation direction, $\tau$ is the delay time, $c$ is the speed of the small signal sound, $\rho_{0}$ is the ambient density, $\delta$ is the sound diffusivity, $\beta$ is the coefficient of nonlinearity, and $\nabla_{\perp}^{2}$ is the transverse Laplacian operator.

The solution to Eq. 1 can be obtained using the method of successive approximations. For simplicity the primary source with Caussian amplitude shading is assumed. By using quasi-linear theory, the solution for the finite-amplitude sound beam generated by the Gaussian source can be expressed as [9]:

$$
q_{1}(r, z)=\frac{p_{0} e^{-\alpha_{1} z}}{1-j z / z_{0}} \exp \left[-\frac{(r / a)^{2}}{1-j z / z_{0}}\right]
$$

where $p_{0}$ is the peak source pressure, $\alpha_{1}$ is the absorption coefficient at primary frequency $\omega, z_{0}=$ $1 / 2 \mathrm{ka}^{2}$ is the Rayleigh distance, and $a$ is the effective source radius. The directivity can be easily obtained as [9]:

$$
D_{1}(k, \theta)=\exp \left[-\frac{1}{4}(k a)^{2} \tan ^{2} \theta\right]
$$

where $k=\omega / c$ is the wavenumber and $\theta$ is the angle with respect to the axis of the beam. Therefore, for a bi-frequency Gaussian source, provided the absorption is neglected, the directivity of the difference frequency is given by the product of the directivity functions of the primary waves [9], i.e.:

$$
D_{-}(\theta)=D_{l a}(\theta) D_{l b}(\theta)
$$

where $D_{l a}(\theta)$ and $D_{l b}(\theta)$ are the directivity for frequency $\omega_{a}$ and $\omega_{b}$, respectively. Consider a group of $M$ weighted primary sources, which are equally spaced with an interelement spacing of $d$ as shown in Fig. 4 . The directivity $D_{l a}(\theta)$ of the weighted primary sources array for frequency $\omega_{a}$ can be written as:

$$
D_{l a}(\theta)=D_{l}\left(k_{a}, \theta\right) H\left(k_{a}, \theta\right)
$$

where $D_{l}\left(k_{a}, \theta\right)$ is the aperture directivity for frequency $\omega_{a}$ and $H\left(k_{a}, \theta\right)$ is the array response which can be expressed as [10]:

$$
H\left(k_{a}, \theta\right)=\frac{1}{M} \sum_{n=0}^{M-1} h_{a n} e^{j n \omega_{a}\left(\tau-\tau_{0}\right)}
$$

where $h_{a n}$ is the nth transducer weighting, and $n=0,1,2, \ldots, M-1 . \tau=d / c \sin \theta$ is the time delay due to the array geometry. $\tau_{0}=d / c \sin \theta_{0}$ is the time delay due to the beam steering angle $\theta_{0}$. In our study the transducer weightings are varied with frequency. Therefore the array response for frequency $\omega_{a}$ can be expressed as:

$$
H\left(k_{a}, \theta\right)=\frac{1}{M} \sum_{n=0}^{M-1} h_{a n}\left(\omega_{a}\right) e^{j n \omega_{a}\left(\tau-\tau_{0}\right)}
$$


Similarly, the directivity for primary frequency $\omega_{b}, D_{l b}(\theta)$, can be written as:

$$
D_{l b}(\theta)=D_{l}\left(k_{b}, \theta\right) H\left(k_{b}, \theta\right)
$$

where $D_{l}\left(k_{b}, \theta\right)$ is the aperture directivity for frequency $\omega_{b}$ and $H\left(k_{b}, \theta\right)$ is the array response which can be expressed as:

$$
H\left(k_{b}, \theta\right)=\frac{1}{M} \sum_{n=0}^{M-1} h_{b n} e^{j n \omega_{b}\left(\tau-\tau_{0}\right)}
$$

where $h_{b n}$ is the nth transducer weighting, and $n=0,1,2, \ldots, M-1$. If the weightings are varied with frequency, the far-field array response can be expressed as:

$$
H\left(k_{b}, \theta\right)=\frac{1}{M} \sum_{n=0}^{M-1} h_{b n}\left(\omega_{b}\right) e^{j n \omega_{b}\left(\tau-\tau_{0}\right)}
$$

Substitution of Eq. 5 and Eq. 8 into Eq. 4 yields

$$
D_{-}(\theta)=D_{l}\left(k_{a}, \theta\right) H\left(k_{a}, \theta\right) D_{l}\left(k_{b}, \theta\right) H\left(k_{b}, \theta\right)
$$

where $D_{-}(\theta)$ is beam pattern for audible frequency, i.e. the difference frequency directivity.

The main objective of the optimization method proposed in the study is to control the beam width of the main lobe and the side lobes' level of the difference frequency directivity in Eq. 13 . The observation points are set from $-40^{0}$ to $+40^{\circ}$ in the study. The formulation of the optimization approach proposed in the work for designing directional audible sound systems can be expressed as:

\section{Minimize $\alpha$}

\section{Subject to}

$$
\begin{aligned}
& \left\|D_{-}\left(\theta_{1}\right)\right\|_{\infty}<\alpha \\
& \left\|D_{-}\left(\theta_{2}\right)\right\|_{\infty}<\alpha \\
& \left\|D_{-}\left(\theta_{3}\right)-D_{-}\left(\theta_{1}\right)\right\|_{\infty}>\delta \\
& \left\|D_{-}\left(\theta_{3}\right)-D_{-}\left(\theta_{2}\right)\right\|_{\infty}>\delta
\end{aligned}
$$

where $\alpha$ is a real number, $\theta_{1}$ is the angle of the side lobe 1 , i.e. $-40^{0}\left\langle\theta_{1}\left\langle-\Delta / 2, \theta_{2}\right.\right.$ is the angle of the side lobe 2, i.e. $\Delta / 2\left\langle\theta_{2}\left\langle 40^{\circ}, \theta_{3}\right.\right.$ is the angle of the main lobe, i.e. $-\Delta / 2\left\langle\theta_{3}\langle\Delta / 2, \Delta\right.$ is the desired beam width, and $\delta$ is the amplitude difference between the main lobe and side lobe.

\section{Results}

In this section the simulated directivity of the audible sound beam created by using the optimization method as shown in Eq. 14 is presented, and then compared to those obtained by using Chebyshev weighting method [5]. In this study, the number of weighting functions in the directional audible sound system is 8 for frequencies $\omega_{a}$ and $\omega_{b}$. The carrier frequency of the ultrasonic transducer array is set as $40 \mathrm{kHz}$. The demodulated signal is at the frequency from $40.5 \mathrm{kHz}$ to $60 \mathrm{kHz}$ with $500 \mathrm{~Hz}$ interval. A total of $M=8$ ultrasonic transducer array is used with inter-element spacing $d=9.7 \mathrm{~mm}$. The effective source radius is set at $\mathrm{a}=3.85 \mathrm{~mm}$ and the speed of sound $c$ is $344 \mathrm{~ms}^{-1}$. The weighting functions are calculated for difference frequency's beam width for $\theta_{-}=20^{\circ}$ and $60^{\circ}$ using the proposed method.

Figs. 1 and 2 show the difference frequency's directivity with $\theta_{0}=0^{0}$, i.e. without beam steering, for $\theta_{-}=20^{\circ}$ and $60^{\circ}$ respectively. Figs. 1(a) and 2(a) are the difference frequency's directivity using Chebyshev weighting method [5], and Figs. 1(b) and 2(b) are the difference frequency's directivity using the optimization method proposed in the paper. From the figures it can be seen that the amplitude in the side lobe using the proposed method is lower than that using Chebyshev weighting 
method. This is because the optimization method tried to find the optimal weighting functions which minimize the sum of the squared amplitude of the side lobe and subject to the amplitude difference between the main lobe and the side lobe. Therefore the optimization method proposed in this paper performs better than Chebyshev weighting method. As can be seen from the figures the beam width can also be controlled using the optimization method. This is because the difference frequency's directivity is the product of two primary frequency's directivities, and its beam width always takes on the narrowest beam width of the two primary waves. It can obviously be seen that a constant beam width is achieved for all frequencies using the proposed method.

\section{Conclusions}

In this paper an optimization method has been proposed for designing directional audible sound system. A uniform linear array composed of eight ultrasonic transducers with different weighting functions was used for generating the audible sound beam. The optimal weighting functions were calculated using the optimization method. The theoretical derivation of the proposed method has been described and some simulation results have also been presented in the paper. The performance of the beam width control using the proposed method has been evaluated. It can be seen that the proposed method could effectively control the beam width of the main lobe and the level of the side lobes for the audible sound beam. It is verified by the results that the lower side lobes level could be obtained by using the proposed method. Therefore the proposed method could better control the beam width and good directivity could also be achieved.

\section{Acknowledgment}

The study was supported by the National Science Council of Taiwan, the Republic of China, under project number NSC-100-2221-E-018-010.

\section{References}

[1] HO Berktay. "Possible exploitation of nonlinear acoustics in underwater transmitting applications,' Journal of Sound and Vibration 2(4), pp. 435-461 1965.

[2] Min Chen, Limei Xu, Dagui Huang, Ying Wang and Xuesheng Li. “'Experimental verification of square rooting algorithm for parametric loudspeaker with a PVDF film transducer,' International Journal of Innovative Computing, Information and Control. Volume 4, Number 8, August, pp. 1877-1888, 2008.

[3] FJ Pompei. "The use of airborne ultrasonics for generating audible sound beams," Journal of Audio Engineering Society 47(9), pp.726-730, 1999.

[4] DI Havelock and AJ Brammer. "'Directional loudspeakers using sound beams,'” Journal of Audio Engineering Society 48(10), pp.908-916, 2000.

[5] J Yang, KS Tan, WS Gan, and J Tian. " Modeling of finite-amplitude sound beams: second order fields generated by a parametric loudspeaker,' IEEE Transactions on ultrasonics, ferroelectrics, and frequency control 52(4), pp. 610-618, 2005.

[6] PJ Westervelt. “Parametric acoustic array,’Journal of Acoustical Society of America 35(4), pp. 535-537, 1963.

[7] M Yoneyama and J Fujimoto. "The audio spotlight: An application of nonliear interaction of sound waves to a new type of loudspeaker design,' Journal of Acoustical Society of America 73(5), pp.1532-1536, 1983. 
[8] WS Gan, Jun Yang, KS Tan, and MH Er. "A digital beamsteerer for difference frequency in a parametric array," IEEE transactions on audio, speech, and language processing 14(3), pp.1018-1025, 2006.

[9] MF Hamilton and DT Blackstock. Nonlinear Acoustics. Academic press, San Diego, 1998.

[10] DH Johnson and DE Dudgeon, Array signal processing. Prentice-Hall, NJ, 1993.

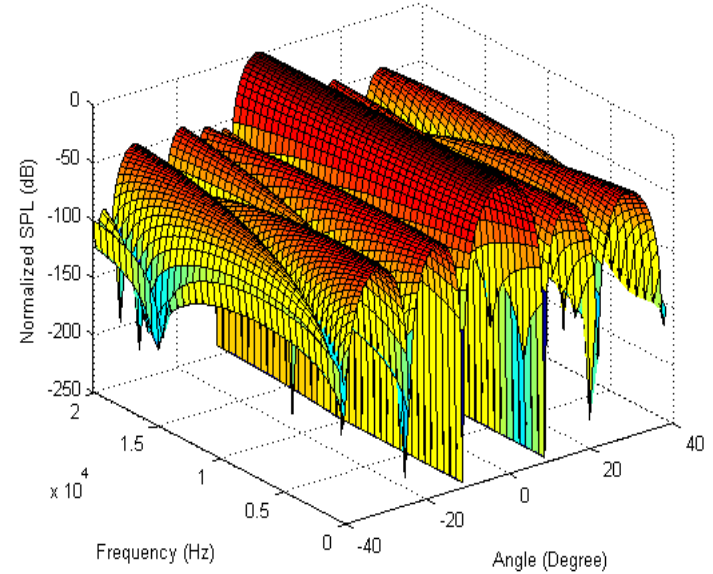

(a)

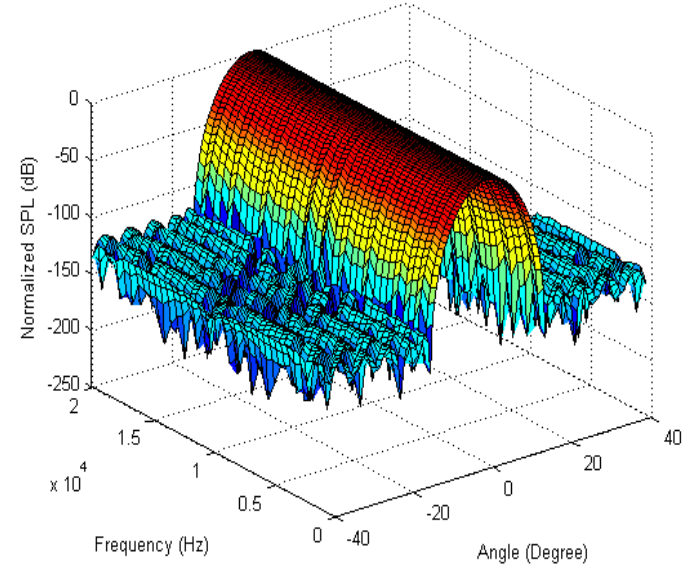

(b)

Figure 1. Difference frequency's directivity for $\theta_{-}=20^{\circ}$. (a) Chebyshev weighting method. (b) Optimization method.

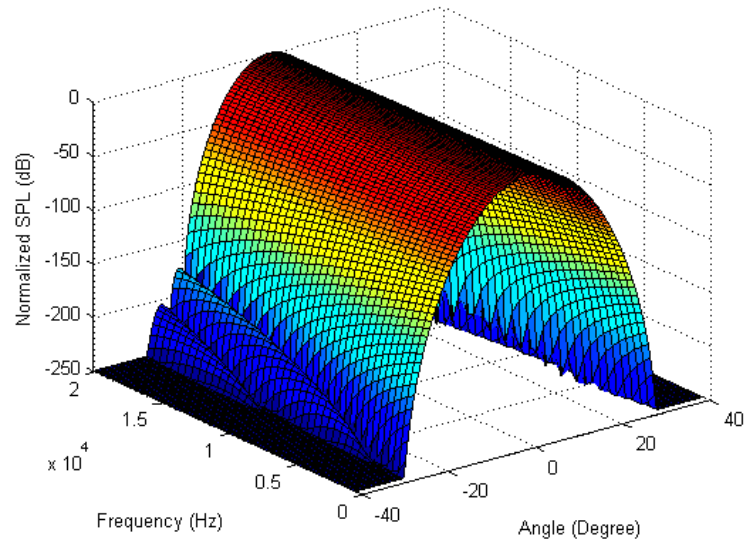

(a)

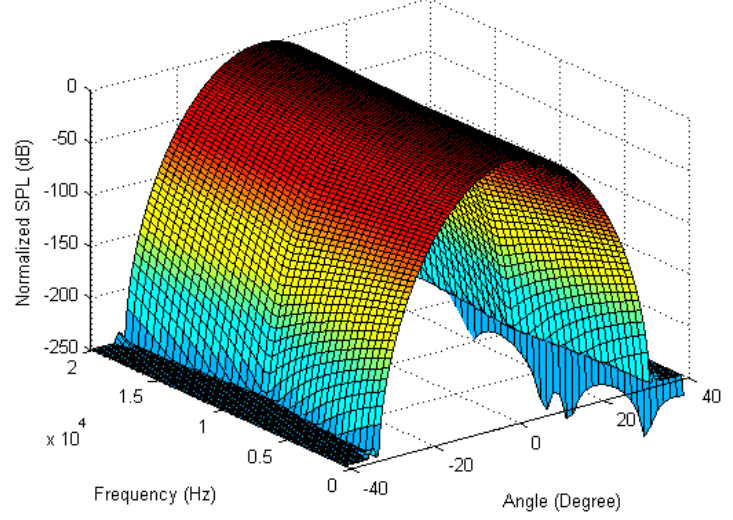

(b)

Figure 2. Difference frequency's directivity for $\theta_{-}=60^{\circ}$. (a) Chebyshev weighting method. (b) Optimization method. 